\title{
An Empirical Study of R Applications for Data Analysis in Marine Geology
} \author{
Polina LEMENKOVA ${ }^{1, *}$ \\ ${ }^{1}$ College of Marine Geoscience, Ocean University of China, Qingdao, China \\ *Correspondence: lemenkovapolina@stu.ouc.edu.cn, pauline.lemenkova@gmail.com
}

Summary: The study focuses on the application of $\mathrm{R}$ programming language towards marine geological research with a case study of Mariana Trench. Due to its logical and straightforward syntax, multi-functional standard libraries, $\mathrm{R}$ is especially attractive to the geologists for the scientific computing. Using R libraries, the unevenness of various factors affecting Mariana Trench geomorphic structure has been studied. These include sediment thickness, slope steepness, angle aspect, depth at the basement and magmatism of the nearby areas. Methods includes using following R libraries: \{ggplot2\} for regression analysis, Kernel density curves, compositional charts; \{ggalt $\}$ for Dumbbell charts for data comparison by tectonic plates, ranking dot plots for correlation analysis; $\{\mathrm{vcd}\}$ for mosaic plots, silhouette plots for compositional similarities among the bathymetric profiles, association plots; $\{$ car $\}$ for ANOVA. Bathymetric GIS data processing was dome in QGIS and LaTeX. The innovativeness of the work consists in the multi-disciplinary approach combining GIS analysis and statistical methods of $\mathrm{R}$ which contributes towards studies of ocean trenches, aimed at geospatial analysis of big data.

Keywords: R, Programming, Statistical Analysis, Mariana Trench, Bathymetry INTRODUCTION

Mariana Trench is the deepest point of the Earth located in the western part of the Pacific Ocean, eastwards to Philippine islands and China (Figure 1). It crosses four tectonic plates: 
Caroline, Pacific, Philippine Sea and Mariana and has unique features in the trench geomorphology, geological structure, adjusting tectonic plates movements, lithological structure and sedimentation processes, briefly discussed below.

\section{Geographic Location}

Mariana Trench belongs to the deepest trenches of the Earth, with maximal depths above 9-11 km, all of which are located in the western half of the Pacific Ocean. The geographic location of the edge of the Mariana Trench along the coasts of the continents or island arcs is explained mainly by the subduction of oceanic tectonic plates at the boundaries of their collision with Mariana, Pacific, Philippine, Caroline tectonic plates (Ishibashi et al. 2015).

The seabed structure of the Mariana Trench is composed in the following way from top to bottom: a sedimentary cover, a basalt of the leitic composition, a complex of parallel dykes of diabases, an isotropic gabbro, a banded gabbro-ultrabasic complex lying on the mantle ultrabasites (Butuzova 2003). There are differences in the velocity of the longitudinal seismic waves in the geological layers of the Mariana Trench structure: water and sedimentary layer have speed of $3.56 .2 \mathrm{~km} / \mathrm{s}$, basaltic layer has speed of $6.5-7.0 \mathrm{~km} / \mathrm{s}$. The layer of gabbro and banded gabbro are located beneath. The boundary of the crust-mantle is made by a sharp increase in the velocities from 7 to $8 \mathrm{~km} / \mathrm{s}$ (the boundary of the Mohorovicic). The upper mantle is the region with the velocities of $8.0-8.2 \mathrm{~km} / \mathrm{s}$ (Garfunkel et al. 1986). Analogues of the oceanic crust on the ground are ophiolites (Figure 2). The average thickness of the crust of this type under the platforms is about $40 \mathrm{~km}$ (Gurevich 1998). The regions with bark continental type in the ocean are fragments of continental mass formed as a result of the formation of the modern ocean floor causing migration and slow movement of the trench (Husson 2012). 


\section{Sedimentation and Lithology}

The lithological structure and sediment thickness are among the other factors affecting Mariana Trench formation. Relatively few other trenches, comparing to Mariana Trench, also formed on the boundaries of the tectonic plates, but usually at a greater distance from the continents in the rift zones. Therefore, the sedimentation processes within Marina Trench are deeply impacted by the location near the rift zones that are mainly associated with the formation of the underwater mountain ranges and spreading. Their expansion in the sides of the neighbouring lithospheric plates is a result of the rise towards Mariana Trench.

The outflow to the surface of the ocean floor of the substance of the upper layer of the earth's mantle. As a result of the unevenness of this process, the transversed faults are formed that have excellent conditions for sedimentation accumulation. According to available geophysical data (Dubinin and Ushakov 2001), the oceanic crust of the tectonic plates surrounding Mariana Trench is composed by a number of layers. Therefore, the impact of the sediment subduction on the trench dynamics is relatively high, which is highlighted by Horleston and Helffrich (2012).

\section{Geomorphology}

The adjusting continental slopes are high, reaching up to several thousand meters and inclining to $3-6^{\circ}$ (in the south-western part of the trench up to $30-40^{\circ}$ ), the upper boundary of which coincides with the edges of the shelf (depths of 150-200 m). Slopes of the passive margins are strongly complicated by the terraces, ledges, marginal plateaus and canyons. Slopes of the margins are steeper, reaching can 5-7 km in height. The geomorphic structure of the Mariana Trench is complicated by the longitudinal ridges, steps, large landslide bodies and ledges. At the base of the continental slope of passive margins formed by the adjacent 
tectonic plates, Philippine Sea, Pacific and Caroline on the south-west, continental foot forms accumulative body. In turn it is formed by the merged cones of the removal and plumes of the suspension flows and submarine landslides with abyssal sedimentation.

Submarine margins of continents, or transition zones depend on the features of the relief and geology. According to them they are divided into two types: the passive ones and the active ones. The first ones include the shelf, that is mainland bank, the slope and the mainland foot. Having complicated relief structure with marginal seas, island arcs and deep-sea gutters, Mariana Trench belongs to the active type of trench. Due to the geodynamic reasons, Mariana Trench motion, migration and upper plate deformation can be described as the result of the response to the mantle flow and the imbalance between the forces exerted by the lower and upper continental plates at the plate interface.

\section{Bathymetry}

The deepest point of the Mariana Trench, the Challenger Deep, is located in the southwest of the trench with maximal depth of $10,984 \pm 25 \mathrm{~m}(95 \%)$ at $11.329903^{\circ} \mathrm{N} /$ $142.199305^{\circ} \mathrm{E}$ (Gardner et al. 2014). North-western Pacific Ocean is especially characteristic for the vast areas of the bottom of the basins occupied by depressions deeper $6000 \mathrm{~m}$ with a special case of Mariana Trench. Despite the extreme bathymetric values of the Mariana Trench at Challenger Deep, its structure has following pattern: a major total area is being occupied by the abyssal depths (3-6 km), while the extreme depths exceeding $6 \mathrm{~km}$, are smaller in comparison to the previous areas (Uyeda and Kanamori 1979).

The abyssal make up relatively small part of the total area of the Mariana Trench, while the second ones cover about two thirds of the whole. Depths of more than $6000 \mathrm{~m}$ are confined mainly to the deepest part of the trench located in the south-western part of Mariana, 
although individual depressions to depths of 6-7 km, rarely up to $7.5 \mathrm{~km}$, occur in the central part of the basins along the trench (Morgan 1974). The typical depths of the ocean floor of the Mariana Trench are 4000-5000 m where mid-ocean ridges and deep-sea basins formed by numerous mountains and depressions are located. The general form of the bathymetric structure of the Mariana Trench is inclined toward the margin plate being connected with the ocean basic zone. It has high and steep island or continental slopes and more low and gentle slopes from the south-eastern side. The main area of the seafloor of the trench has prevailing depths of more than $3000 \mathrm{~m}$, a seabed being the most ancient parts of the ocean floor formed in the late Jurassic.

\section{MATERIALS AND METHODS}

Various methods have been developed for application at research of ocean trenches. using not only GIS, but also statistical methods and approaches made by programming (e.g. Warner et al. 2008, Oliphant 2007, Roberts et al. 2010). Recently developed powerful technologies provided by $\mathrm{R}$, Matlab or Octava software, enable to perform precise computations and statistical analysis of big data as well as to create data frames in geosciences. Among others, a python languages has become increasingly popular (Lin 2008, Marta-Almeida et al. 2011). Machine learning and data mining for oceanological research is nowadays one of the most important technical goals. Using R programming based machine learning algorithms ensure the preciseness and objectiveness of the big data set processing, which is always the case for oceanographic research.

However, a combination of the statistical methods with GIS dramatically increases the effectiveness of the research due to the embedded machine learning algorithms that enable to process big data frames dividing them into compatible data sets for statistical analysis. For 
instance, we can select several attribute tables specifically for magmatism of the nearby areas to analyse the possibilities of the earthquakes, or to focus on the bathymetric properties and distribution of various depth values across the selected profiles, etc. Special packages of the R programming include mathematical algorithms and a range of codes specially designed for statistical analysis, as demonstrated below. In the scope of current research a functionality of $\mathrm{R}$ language has been tested. Indeed, it proved to be as an effective tool for studying distribution of the environmental factors affecting the structure of the trench, as well as bathymetric clusters grouped by the similarity of the geomorphic properties at the seafloor basement of the Mariana Trench.

The methodological workflow of the current work includes following steps:

1. Bathymetric GIS Data Processing in QGIS and LaTeX;

2. Statistical analysis on data distribution: Kernel density and regression analysis with 3 approaches: linear, "loess" and general linear methods;

3. Dumbbell charts for data pairwise comparison by tectonic plates;

4. Ranking dot plots for correlation between the determinants (trench slope steepness in $\operatorname{tg}^{\mathrm{o}}$ angle by bathymetric profiles, igneous volcanic areas, depth);

5. Compositional "waffle" charts showing variation of the aspect and steepness classes of the trench basement angle, by 25 bathymetric profiles;

6. Mosaic plots with standardised residuals to assess data distribution and silhouette plot for compositional similarities among the bathymetric profiles;

7. Association plots with statistical Pearson residuals to analyse model fitness at each observation data set for generalized linear models;

8. ANOVA (Analysis of Variance) to assess hypothesis testing. 


\section{Bathymetric GIS Data Processing}

The GIS based digitizing of the 25 bathymetric profiles across Mariana Trench has been performed in QGIS. The length of each profile was taken $1000 \mathrm{~km}$, and the distance between every pair was $100 \mathrm{~km}$. The coordinated were saved in a table with three columns: elevations, latitude and longitude. After a square of the area was crossed by the profiles, the .csv table was imported for processing in LaTeX. The visualization of the 25 profiles grouped by three and four has been performed in LaTeX (Figure 3). Technically, the following script was used:

lbegin $\{$ filecontents* $\}\{$ MyTab18.csv $\}$

ELEV ,y2,x2

145.528246366,47.0433461696,-7800 \# bathymetric data here in 3 columns

lend $\{$ filecontents* $\}$

lbegin $\{$ tikzpicture $\}$

lbegin $\{$ axis $\}[$ grid=major,minor $\mathrm{x}$ tick num=10,minor $\mathrm{y}$ tick num $=10$, colorbar sampled line,colormap name $=$ bluered,title $=\{$ Mariana Trench. Bathymetric Profiles

Nr.16,17,18 $\},$ ylabel $=\{$ Depth $(\mathrm{m})\}$, legend entries $=\{$ Profile18,Profile17,Profile16, $\}$, scaled ticks $=$ false, yticklabel style $=\{$

/pgf/number format/fixed,

/pgf/number format/fixed zerofill,\}]

laddplot+ [scatter,only marks,mark=Mercedes star flipped,colormap name=bluered,] table $[\mathrm{x}=\mathrm{x}, \mathrm{y}=\mathrm{d}$, col sep=comma $]\{$ MyTab16.csv $\}$;

laddplot+ [scatter, colorbar sampled line,only marks,mark=asterisk,colormap name $=$ bluered, $]$ table $[\mathrm{x}=$ long, $\mathrm{y}=\mathrm{d}$, col sep=comma $]\{$ MyTab17.csv $\}$; 
laddplot+ [scatter, colorbar sampled line,only marks,mark=10-pointed star,colormap name=bluered, $]$ table $[\mathrm{x}=\mathrm{y} 2, \mathrm{y}=\mathrm{ELEV}, \mathrm{col} \mathrm{sep}=\mathrm{comma}]\{$ MyTab18.csv $\}$;

lend $\{$ axis $\}$ lend $\{$ tikzpicture $\}$

\section{Regression Analysis of Data Distribution: R library \{ggplot2\}}

Hence, depending on the structure and size of the availability data frame, the methodological approaches of the assessment of data distribution may change. However, the most essential statistical analysis of the ocean research would start by the question of data distribution. Computing and plotting Kernel distribution curves, box plots, regression lines (probability of bathymetric data across profiles), quantile statistics, empirical distribution density function and other methods can be distinguished as the most useful for initial data processing.

The principle of the regression analysis is based on the analysis of the probability of the data values according to their actual distribution. A regression analysis represents outlying depths observations by each bathymetric profile, as shown on the example of three profiles in Figure 4. This methodology utilizes the relation between two or more quantitative depth variables so that one variable can be predicted from another. Thus, one can estimate the probability that the depth values will be located in this or that interval of values profile by profile according to the machine-based algorithm of the probability of their distribution. Kernel density distribution shows the majority of the depths of the trench between 3000 and $6000 \mathrm{~m}$. The graph has been made as a combined plot (Figure 5) enabling to compare the overlapping and maximal aptitude of the density curves both for the profiles and depth data distribution by four tectonic plates.

\section{Dumbbell Charts for Data Pairwise Comparison by Tectonic Plates}


Because Mariana Trench crosses four tectonic plates (Mariana, Caroline, Philippine and Pacific) the comparative testing of the values distribution by adjacent tectonic plates has been completed through the Dumbbell chart plotting. Dumbbell chart is a visualization aimed to give an insight of how the margin tectonic plates constitute to the Mariana Trench pairwise. It is one of the new statistical methods that initially was widely used in biosciences, yet can be applied to spatial analyses when it is necessary to take pairwise comparison of the data distribution from any thematic layer.

Plotting has been carried out by calling R libraries $\{$ ggplot 2$\}$ and $\{$ ggalt $\}$. Dumbbell chart demonstrates (Figure 6) pairwise distribution of the bathymetric points constituting the continental plates, to show the composition of the sedimentary coverage by four tectonic plates. The $\mathrm{Y}$ axis shows the profiles and the $\mathrm{X}$ axis - the number of observation points crossing to the given tectonic plate. In this cases the comparison was given by pairs Philippine sea and Pacific plate, and Mariana and Caroline Plates, respectively. Although a complete comparison of al the environmental determinants has not yet been given (in the scope of this research only bathymetric points were plotted for the Dumbbell chart), a certain insight of the tectonic structure of the Mariana Trench contributes to understanding of the congruence of the ocean floor by four plates.

\section{Ranking Dot Plots by Data Grouping}

Pairwise correlation was visualized by the ranking dot plots, which is effective tool to perform data grouping by variables: tectonic plates. Plotting distribution of the observation points by igneous volcanic areas aimed at visualizing areas affected by the magmatism. Large igneous volcanic areas contribute towards localization of possible earthquake zones. Thus, as can be drawn from the plot (Figure 7), the volcanic areas increase along Mariana Trench 
south-westwards. Alike to the volcanic areas plotting, the variation of the steepness slope was performed using ranking dot plot: the bathymetric profiles with the most steep angles across the trench are located in the north-eastern part with a slight decrease towards the south-west. To calculate $\operatorname{tg}^{\mathrm{o}}$ angle of the profiles, a standardized formula was used, that is a relation of the maximal depth by profiles divided by the width of the corresponding bathymetric profile. The crucial points (Figure 7) were selected using library\{ggalt $\}$ by calling following R code:

crucial_igneous $<$ MDF[MDF\$igneous_volc $>50 \&$ MDF\$igneous_volc $<=300 \&$ MDF\$profile $>5 \&$ MDF\$profile $<=25$,

crucial_angles <- MDF[MDF\$tg_angle $>0.00 \&$ MDF\$tg_angle $<=0.075 \&$ MDF\$profile $>5 \&$ MDF\$profile $<=22$,

The distribution of angle steepness values across the trench have been shaped by the components of the igneous volcanic areas near the profiles, as understood from the Figure 7: profiles \# 20, 22, 24, 8 and 11 with notable amount of igneous volcanic observation points (over 180) correlate with steepness angle.

\section{Compositional charts of the determinants variations}

The constitution of the system composition has been visualized by the categorical plotting. One of the methods enabling categorical plotting is presented by compositional charts, sometimes referred to as "waffle charts". The core idea of this method is to demonstrate the division of the whole system by parts in percentage. Several R libraries can be used to perform technically compositional plotting, of which the $\{$ ggplot 2$\}$ has been selected as the most effective: it enables to control the appearance of the plot by adjusting details (colors, font size and types, plotting two graphs together, etc). The compositional chart is aimed to compare the distribution of the data across the study area, as well as the 
composition of the aspect class and slope angle for the Mariana Trench. As can be drawn from the chart (Figure 8), the category "very steep slope" is the dominant among all other geomorphological types of the slope degree of the Mariana Trench. Likewise, north and south-west aspect of the slope direction are best describing the geometry of the northern and southern part of the trench, respectively.

\section{Mosaic and silhouette plots of the observation data by tectonic plates}

Mosaic chart aims at categorial comparison of the geomorphological features of the trench by four tectonic plates. Visualizing mosaic plot is a statistical method that involves a subdivision of a rectangular tiles into the areas that represent the conditional relative frequency for a cell in the contingency table. The algorithms and approaches used in mosaic plotting vary slightly. Upon examination of possible packages, the R library $\{\mathrm{vcd}\}$ was selected for this research. Using $\{\mathrm{vcd}\}$ library, each tile is colored to show the deviation from the expected frequency, that is residuals, from a Pearson $X^{2}$ or likelihood ratio $G^{2}$ test. The algorithm set includes execution of the following code:

MosaicTectRes<- mosaicplot $($ count, main = "Mariana Trench: InMosaic Plot", sub = "1:518 observation points in each profile", xlab = "Tectonic Plates", ylab = "Profiles", las = 1, border $=$ "chocolate", shade $=$ TRUE)

The convenience of the mosaic plot for the geomorphological analysis of the ocean trenches consists in its visual representation of the association between the environmental variables. Thus, it gives am overview of the data structure and enables to recognize relationships between the different environmental variables. As can be drawn from the Figure 17, the independence of the Caroline tectonic plate is shown clearly. Conversely, the boxes across categories constituting Pacific and Mariana plates have similar areas. The area of the 
tiles, the bin size, includes the identification of the sampling data (518 observation points across 25 bathymetric profiles), giving the proportional value to the number of observations within that category.

The interpretation and validation of consistency within clusters of geomorphologic and geological variables have beet tested using silhouette method (Figure 9). This technique provides a succinct graphical representation of the suitability and fitness of each from 518 observation points across 25 bathymetric profiles within the clusters. The silhouette value measured similarity of the points derived from the thematic layers (sediment thickness, slope steepness of the trench) to the cluster by cohesion and comparison them with other clusters, and separating them from the distinct points.

Thus, lower sedimental thickness area would lie within one class while a class of areas with high level of sedimental level fits to another. The silhouette ranges from -1 to +1 (Figure 9), where a high value indicates that the areas of trench are well matched to their clusters and poorly matched to the neighboring ones. To achieve the sufficient and appropriate configuration of the chosen cluster plotting, the geomorphic groups were grouped by values. The silhouette was calculated with Euclidean distance metric by calling library\{cluster\} using following R code:

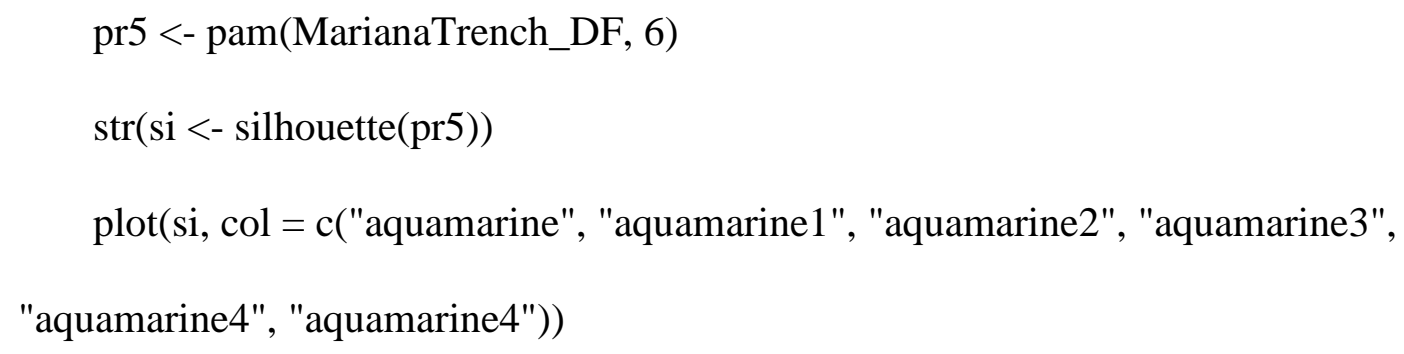

The given script plots the silhouettes, returns the values in the n-by- 1 vectors.

\section{Association plots with statistical Pearson residuals}


A deviations from the independence of rows and columns containing bathymetric data and environmental variables is presented in a two-dimensional contingency table of the Cohen-Friendly association plots (Figure 10). Extended association plot in $\mathrm{R}$ reveals relationships between the variables by the assoc() function in the $\{\mathrm{vcd}\}$ package. In this case, it is tectonic plates and geomorphological values (bathymetric depths, slope steepness). Pearson's residuals on the association plot report test for the statistical goodness of fit: it enables to assess the model fit at each of 518 observation for every bathymetric profile for the generalized linear models within the context of the chi-square (Figure 10). It has been computed as a raw residual divided by the square root of the variance function. Technically, association plot method includes execution of the following $\mathrm{R}$ code:

library(vcd) \# calling necessary package

MDF <- read.csv("Morphology.csv", header=TRUE, sep = ",") \# uploading table

MDF <- na.omit(MDF) \# deleting non available values in the data frame

row.has.na <- apply(MDF, 1 , function(x) $\{\operatorname{any}($ is.na(x) $)\})$

sum(row.has.na) \# checking up non available values in the data frame

head(MDF) \# visualizing data frame as a table

\# step-2. Merging columns in a table according to their category values (tectonic plates)

MDTt $=$ melt $($ setDT $($ MDF $)$, measure $=$ patterns $(" \wedge$ plate" $)$, value.name $=c("$ tectonics" $))$

levels(MDTt\$variable) = c("Philippine Plate", "Pacific Plate", "Mariana Plate", "Caroline Plate")

assoc(count, shade=TRUE) \# plotting association plot according to the tectonic plates crossing the Mariana Trench.

\section{Analysis of Variance (ANOVA) to assess hypothesis testing}


The results have been interpreted on the homogeneity of variance by means of the oneway ANOVA series of tests (Figure 11). Several test methods were executed in this research. The brief description of their advantages and particularities is given below. The Tukey HSD (Tukey Honest Significant Differences) multiple pairwise-comparisons was applied to the ANOVA results. Since the ANOVA test is significant, the Tukey HSD test was computed by R function. TukeyHSD() takes the fitted ANOVA as an argument. This test enabled to perform multiple pairwise-comparison between the means of groups. The library\{multcomp\} was used to perform a Pairewise t-test and to calculate pairwise comparisons between the group levels with the corrections for the multiple testing using $\mathrm{R}$ function pairewise.t.test(). The Levene's test was used to analyse the homogeneity of variances at the next step of ANOVA testing by calling function leveneTest () in the $\{$ car $\}$ package. Comparing to the Pairewise t-test, the advantages of the Levene's test is its lesser sensitiveness towards the departures from the normal distribution. Afterwards, the Welch test was executed in parallel to the oneway.test() in experimental mode. The Welch-test does not require that assumption have been implemented in the function oneway.test() which make is more suitable comparing to the last one. The Shapiro test was alternatively used to analyse variance of the residuals (Figure 19, upper left). Finally, a non-parametric Kruskal-Wallis rank sum test was applied to finish with a series of ANOVA testing. The result have been interpreted on homogeneity of variance by means of one-way ANOVA tests.

Since the p-value (Figure 11) is less than the significance level 0.05, one can conclude that there are significant differences between the groups highlighted with "**" in the model summary, that is four tectonic plates: Philippine, Pacific, Mariana and Caroline. The Tukey multiple pairwise-comparisons test enabled to perform multiple pairwise-comparison between 
the means of groups and demonstrated $95 \%$ family-wise confidence level of the results. The plotted homogeneity of variances of the results are shown in Figure 11.

\section{RESULTS}

As can be drawn from the Figure 5, Mariana plate has the highest Kernel density of depth distribution values, followed by the Philippine plate, then Pacific and Caroline, respectively. Kernel density distribution has been shown in a combined plot enabling to compare the overlapping and maximal aptitude of the density curves both for the profiles and depth data distribution by four tectonic plates. The major trend of the trench angles located on the Pacific plate has downward general line trend. The Philippine tectonic plate, on the contrary, has a minimal peak by profiles \#14-21, and then moving upwards. The highest value for Caroline plate has profile \#23, while the maximal level for Mariana plate has profile \#7. The Philippine tectonic plate, on the contrary, has a minimal peak by profiles \#14-21, and then moving upwards. The highest value for Caroline plate has profile \#23, while the maximal level for Mariana plate has profile \#7.

From the composition charts (Figure 8), drawn to compare the slope angles and aspect degree by bathymetric profiles, one can see the unique patters of these categories for the trench. The basic concepts of the close interrelation of various environmental factors (geological structure, bathymetric patters, tectonic plates location and subduction, spreading, transform fault, depths) affecting Mariana Trench formation is reflected in this paper. The technical tools consist of combination of $\mathrm{R}$ programming, GIS spatial analysis and mathematical algorithms for data processing and modelling. A comparison of the properties of the oceanic crust and ophiolite sections, sedimentary thickness and depth distribution across bathymetric profiles enabled to make a better understanding of the ties among geospatial 
factors influencing Mariana Trench formation. In turn, it facilitate a more reasonable forecast of the possible position of the mineral deposits fossils within Mariana Trench, for instance in the Philippine Sea tectonic plate.

The algorithm based objective analysis of the Mariana Trench bathymetric unevenness enables to better understand its morphology and structure. The special section of this research is focused on the detailed technical evaluation of the R and LaTeX scripts and description of their functionality. The thorough description of the workflow steps is accompanied by the written most important $\mathrm{R}$ scripts and codes provided in full, what makes this work repeatable. The discussion of the advantages of the methods enables to have a representative information on the key procedures of the research. As has been demonstrated in this paper, the combination of the statistical analysis by $\mathrm{R}$ programming language, modelling algorithms and GIS geospatial analysis provides the most effective methods for studying ocean floor and hadal trenches, the least reachable research objects on the Earth.

The study of the structure of the Mariana Trench has a great scientific and practical interest. The first relates to the testing theory of plate tectonics. The second is caused by the estimation of the time and place of where earthquakes and tsunami may arise. Moreover, a correlation with distribution of the volcanic igneous areas, as well as economic needs for the mineral resources are other important goals for deep trench studies. The aim of the presented research was to identify bathymetric cross section profiles of the Mariana Trench that show similarity by their complex parameters, so that they could be grouped into patters. Using combination of R programming, LaTeX visualization and GIS, supported by the traditional geologic research, this study enabled to identify variabilities among factors affecting Mariana trench morphology. The accuracy of the performed statistical measurements is impacted by 
the chosen methodology and the input data: geomorphological maps, geological attribute tables, bathymetric maps, other geospatial thematic layers.

\section{DISCUSSION}

The question of the formation and structure of the ocean trenches has long attracted oceanographers. A variety of research work has been performed applied for various aspects of the hadal trenches: to measure trench depth, to assess the volumes of possible hidden resources in the abyssal depths, to analyse pelagic and biotic communities, to predict earthquakes frequencies. Nowadays, sound statistical methods are elaborated as important additions for traditional GIS methods of geospatial analysis of the deep ocean trenches. Without machine learning algorithms, a geospatial analysis of the big data cannot be considered as accurate and may lead to minor or major shortcomings and errors. Machine learning is therefore indispensable for such remotely located areas as abyssal depths.

The principle of $\mathrm{R}$ based geospatial data mining for the ocean research is to perform fusion and integration of multi-source data that allows integration of the heterogeneous information from a variety of sources for the possibility of in-depth analysis. In such a way, a multi-dimensional data processing is possible including following thematic layers: tectonic (continental margin plates), geological (geologic structure), lithological (sedimentation) and oceanological (direction of the deep currents). Automatic reflection of the multi-variant data by the machine learning methods enables to speed up our understanding of the oceans, as well as to increase the accuracy of the research.

The paper presents an application of the $\mathrm{R}$ language towards geological studies with a specific case of marine geological features, such as Mariana Trench. The brief sketch on the structure of the main environmental factors of the Mariana Trench has been presented in 
introduction section, briefly summarizing tectonic plates movements, geological structure, sedimental conditions, bathymetry, lithological properties and other features of the Mariana Trench system. It furthermore reviews the impact of the environmental determinants on the Mariana Trench formation and structure, for instance, the relationship of the transform faults with rift valleys, location of the large igneous polygons. The methodology chapter is focused on the technical approaches of the study of the trench morphology by means of $\mathrm{R}$ programming, statistical analysis and GIS. The techniques of the classification are presented, including key R programming scripts. The results chapter highlights the findings received by the application of the technical methods (R programming, statistical algorithms and GIS) with geological analysis of the Mariana Trench seafloor. The graphical and cartographic materials presented in this research support results and findings. Current work demonstrated that combination of $\mathrm{R}$ language, statistical algorithms and QuantumGIS is a highly effective decision for geospatial research aimed at processing multi-dimensional geospatial data sets.

As a recommendation for further research focused on ocean trenches, a traditional methods of the seafloor studies at the GIS level should always include a set of statistical analysis tools such as R, or alternatively, python (e.g. ScyPy, NumPy, matplotlib), Matlab, Octave. Studies of the spacial data series are usually accompanied by the graphics in view of the facetted plots that can facilitate comparison of the data by groups. This is especially useful while collecting multi-dimensional various geophysical data. Since there are many deep interconnections among factors affecting the formation, morphology and development of the trench, mathematical algorithms and objective analyses based approaches are to be used to study marine geo-systems.

\section{ACKNOWLEDGEMENTS}


The funding of this research has been provided by the China Scholarship Council (CSC) State Oceanic Administration (SOA), Marine Scholarship of China [Grant \# 2016SOA002, 2016].

\section{REFERENCES}

Butuzova G.O. 2003. Hydrothermal-sedimentary ore formation in the world the ocean M. GEOS, 136 pp. (in Russian: Бутузова Г.Ю. Гидротермально-осадочное рудообразование в Мировом океане. ГЕОС, Москва, 2003 г., 136 стр.)

Dubinin E.P., Ushakov S.A. 2001. Oceanic rift genesis. M. GEOS, 293 pp. (in Russian: Дубинин Е.П., Ушаков С.А. Издание: ГЕОС, Москва, 2001 г., 293 стр.)

Gardner J.V., Armstrong A.A., Calder B.R., Beaudoin J. 2014. So, how deep is the Mariana Trench? Mar. Geodesy, 37(1): 1-13.

Garfunkel Z., Anderson C.A., Schubert G. 1986. Mantle circulation and the lateral migration of subducted slabs. J. Geophys. Res. 91: 7205-7223.

Gurevich E.G. 1998. Metalliferous sediments in the World Ocean. M: Nauchnuy Mir, 340 pp. (in Russian).

Horleston A.C., Helffrich G.R. Constraining sediment subduction: A converted phase study of the Aleutians and Marianas. Earth and Planetary Science Letters 359-360 (2012) $141-151$.

Husson L. 2012. Trench migration and upper plate strain over a convecting mantle. Phys. Earth Planet. Int. 212-213: 32-43.

Ishibashi J., Tsunogai U., Toki T., Ebina N., Gamo T., Sano Y., Masuda H., Chiba H. 2015. Chemical composition of hydrothermal fluids in the central and southern Mariana Trough backarc basin. Deep-Sea Res. II 121: 126-136. 
Lin J.W.B. 2008. qtcm 0.1.2: a Python implementation of the Neelin-Zeng quasiequilibrium tropical circulation model. Geosci. Model. Dev. 1: 315-344.

Marta-Almeida M., Ruiz-Villarreal M., Otero P., Cobas P., Peliz A., Nolasco R., Cirano M., Pereira J. 2011. OOF3: A Python engine for automating regional and coastal ocean forecasts. Env. Model. Software, 26: 680-682.

Oliphant T.E. 2007. Python for scientific computing. Comput. Sci. Eng. 9: 10-20.

Roberts J.J., Best B.D., Dunn D.C., Treml E.A., Halpin P.N. 2010. Marine geo-spatial ecology tools: an integrated framework for ecological geoprocessing with ArcGIS, Python, R, MATLAB, and C++. Env. Model. Software 25: 1197-1207.

Uyeda S., Kanamori H. 1979. Back-arc opening and the mode of subduction. J Geophys Res 84: 2017-2037.

Warner J.C., Perlin N., Skyllingstad E.D. 2008. Using the model coupling toolkit to couple earth system models. Env. Model. Software 23: 1240-1249. 
Mariana Trench: enlarged study area

Cartographic visualization: Quantum GIS 3.0

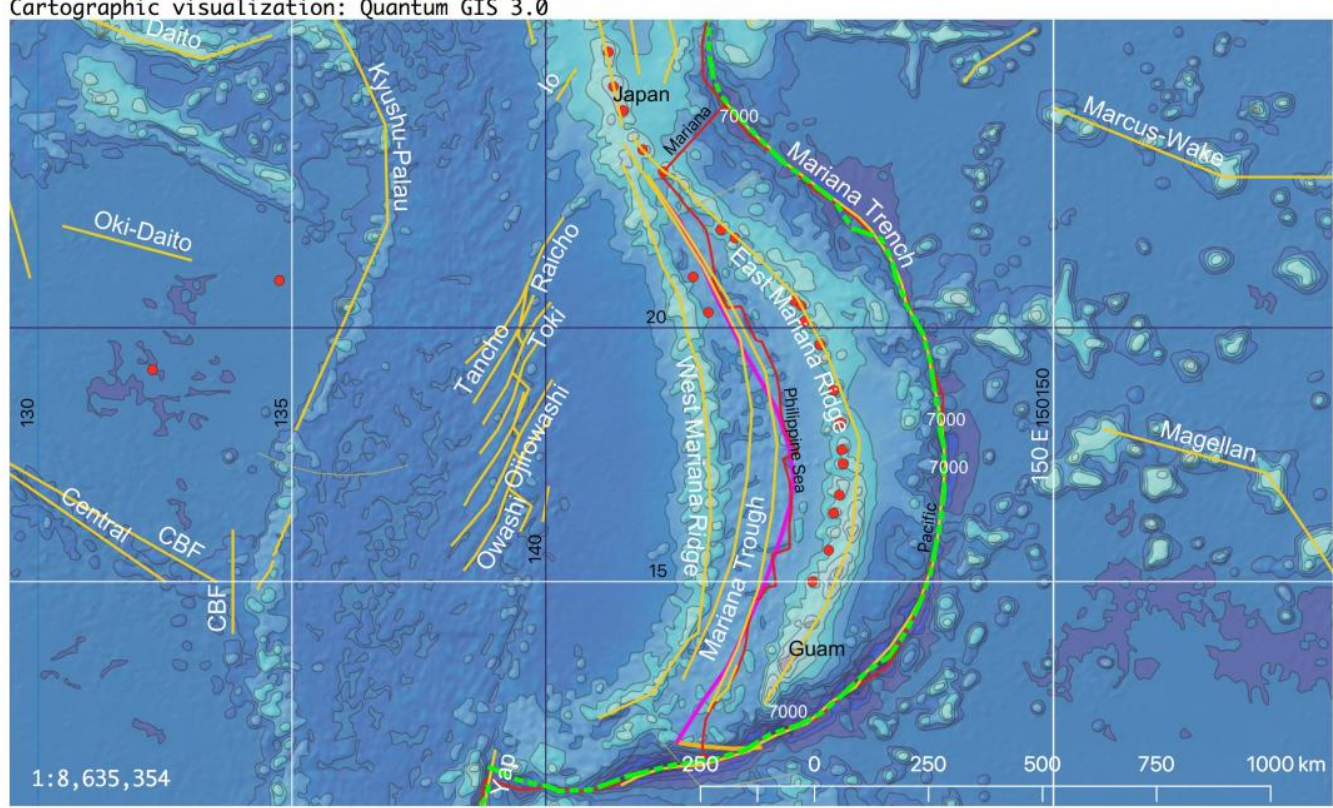

Legend

-- trench - Transform lines bathymetry $10000 \quad \square$ bathymetry_8000 $\square$ bathymetry_5000 $\square$ bathymetry_2000 $\square$ bathymetry_0

— Fault lines $\quad$ Volcanoes hotspots $\square$ bathymetry $10000 \square$ bathymetry_7000 $\square$ bathymetry_4000 $\square$ bathymetry_1000

- Ridge lines $\quad$ bathymetry_9000 $\square$ bathymetry_6000 $\square$ bathymetry_3000 $\square$ bathymetry_200

Figure 1. Study area: Mariana Trench. Cartography by QuantumGIS (QGIS) 
Geological map for the "Mariana Trench" GIS project. Lithology around the study area. Visualization: QGIS.

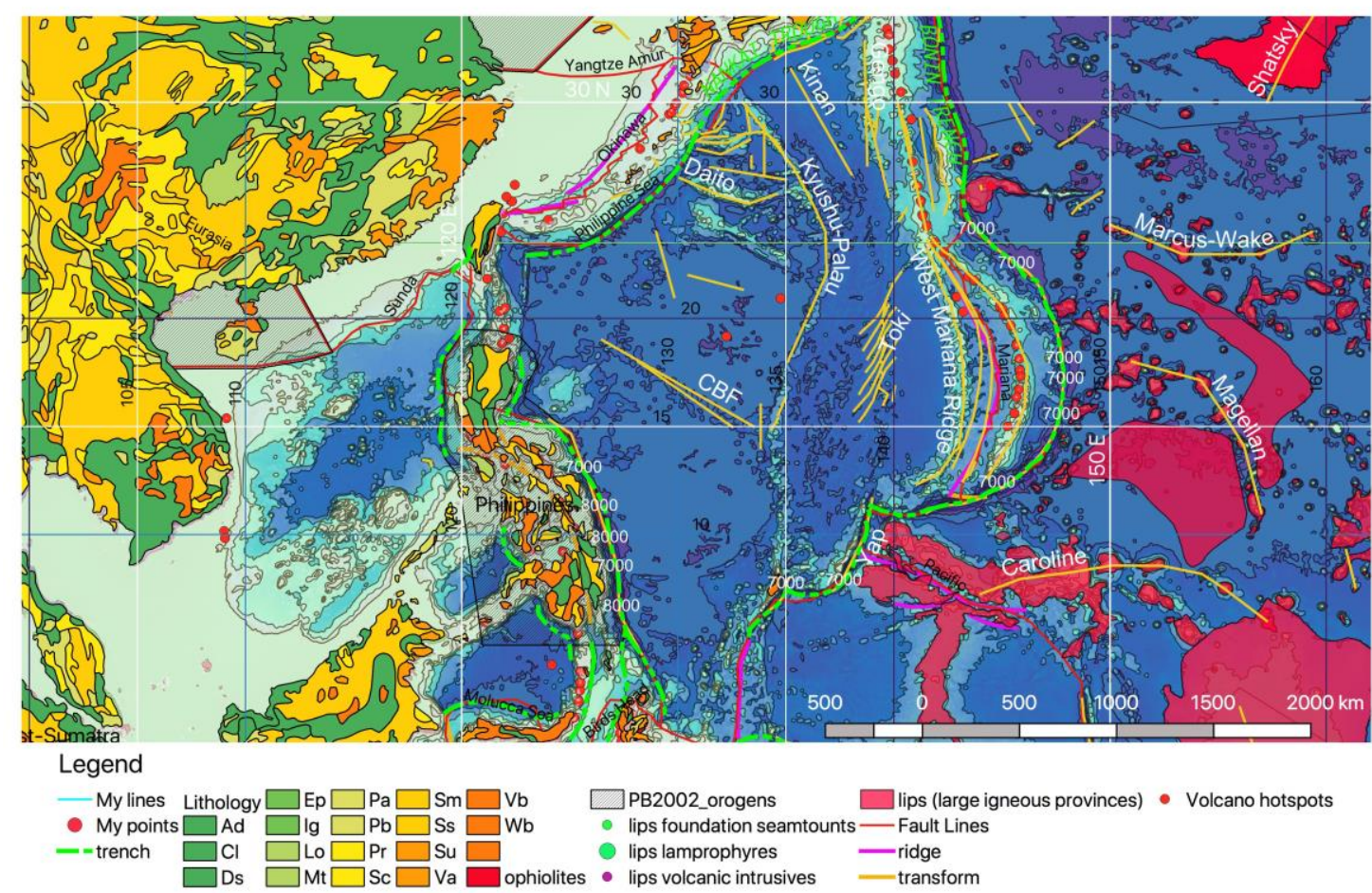

Figure 2. Lithology of the Mariana Trench area, Pacific Ocean. Map: QGIS 

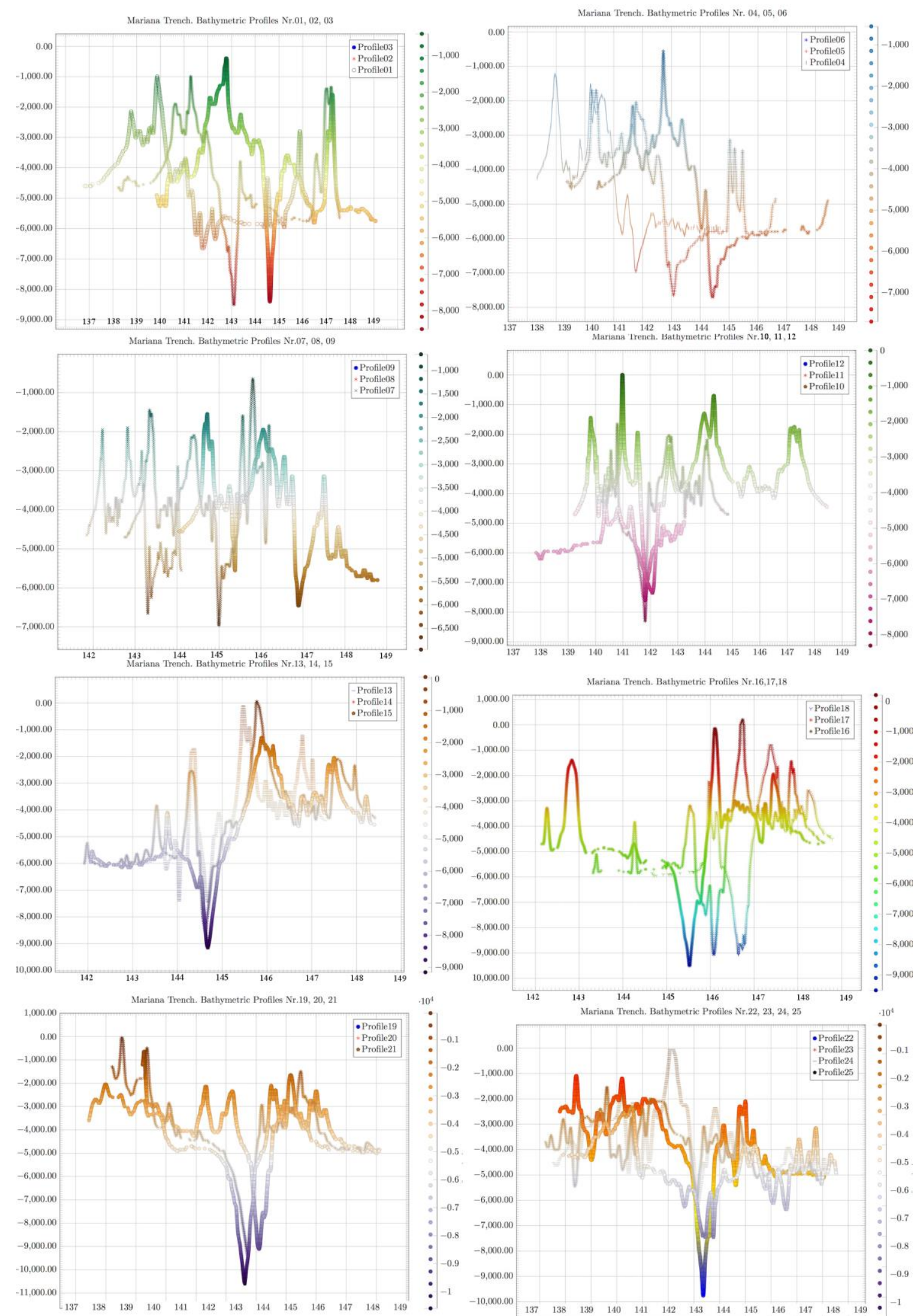

Figure 3. 25 cross-section bathymetric profiles of Mariana Trench. Plotting: LaTeX 
1 马里亚约海沟, 剖面11，Mariana Trench, Profile Nr.11.

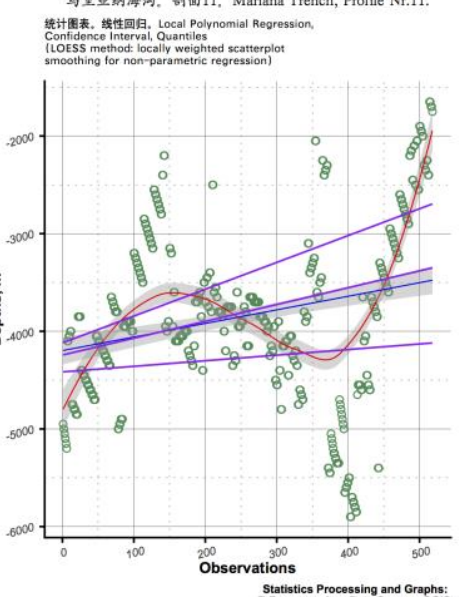

2 马里亚结海沟, 剖面18, Mariana Trench, Profile Nr.18.

3 马里亚络海沟, 新面24, Mariana Trench, Profile Nr.24.

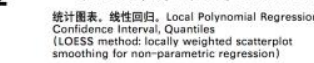

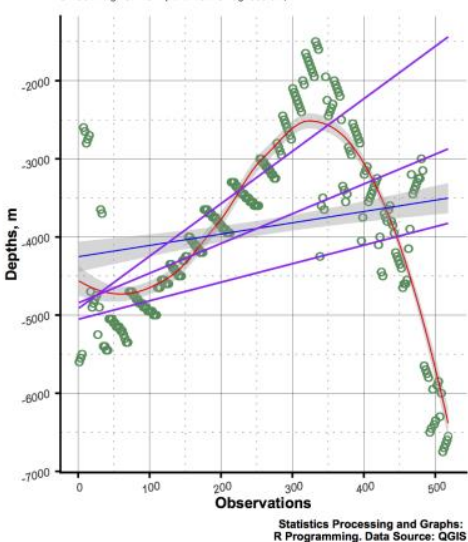

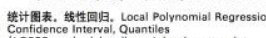

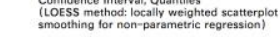

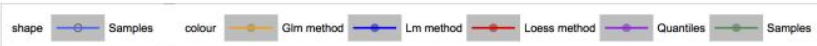

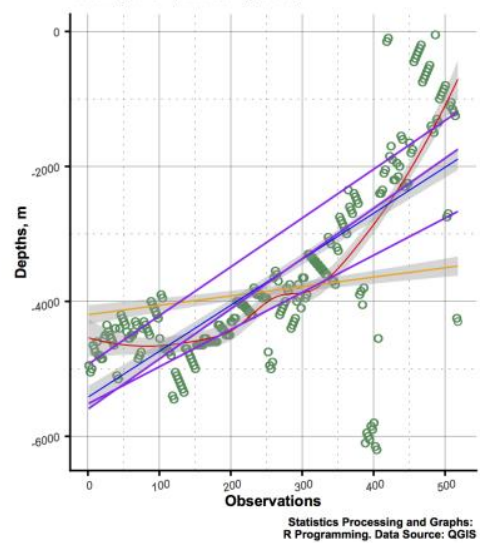

Figure 4. Regression analysis for selected profiles, R Programming 

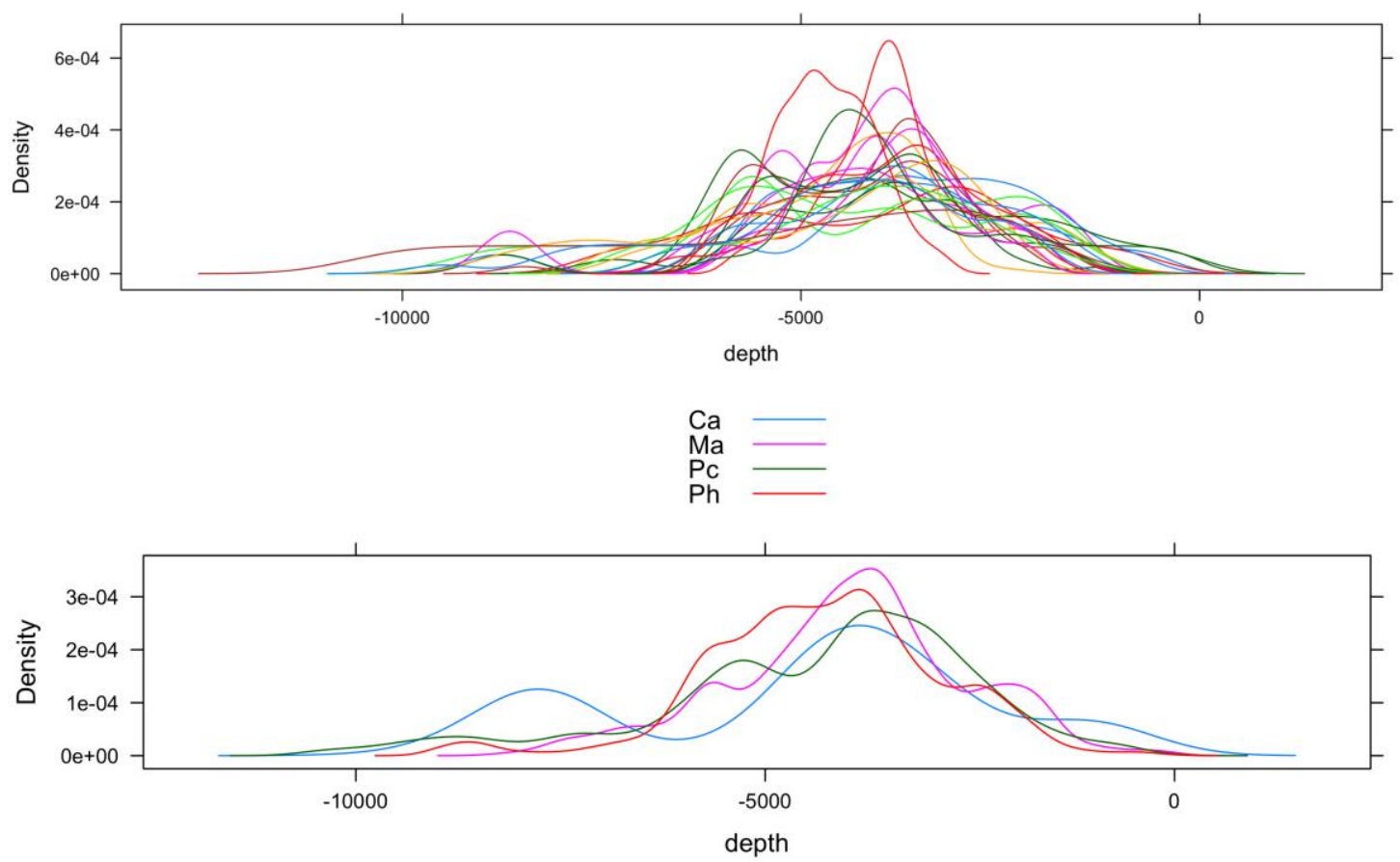

Figure 5. Kernel density estimation for 25 bathymetric profiles, Graph: R. 
1 马里亚纳海沟。剖面1-25。 Dumbbell Chart Mariana Trench, Profiles Nr.1-25.

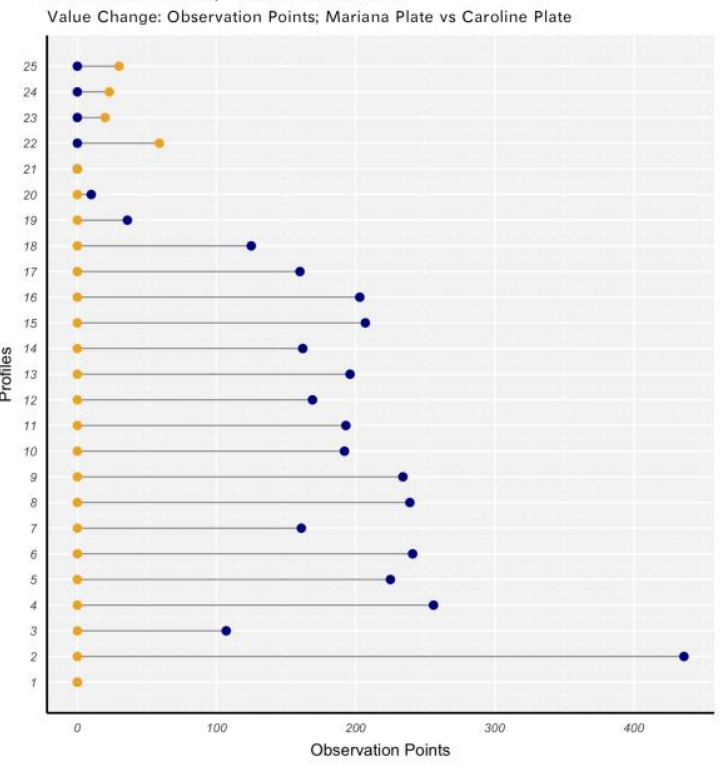

海洋大学, 青岛市2018.
Statistics Processing and Graphs: R Programming. Data Source: QGIS
2 马里亚纳海沟。剖面1-25, Dumbbell Chart Mariana Trench, Profiles Nr.1-25.

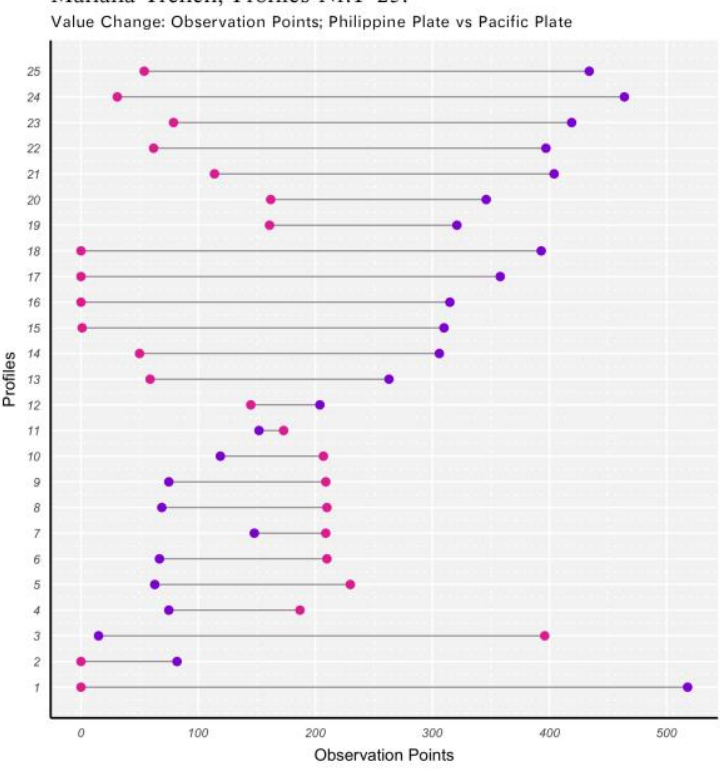

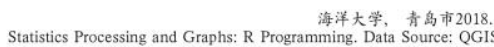

Figure 6. Dumbbell diagrams for pairwise comparison of the tectonic plates 
1 马里亚纳海沟。剖面1-25。 Mariana Trench, Profiles Nr.1-25. 统计图表。地貌聚类分析, 圆点图。Ranking Dot Plot. 统计图表.
Distribution of Observation Points across Igneous Volcanic Areas

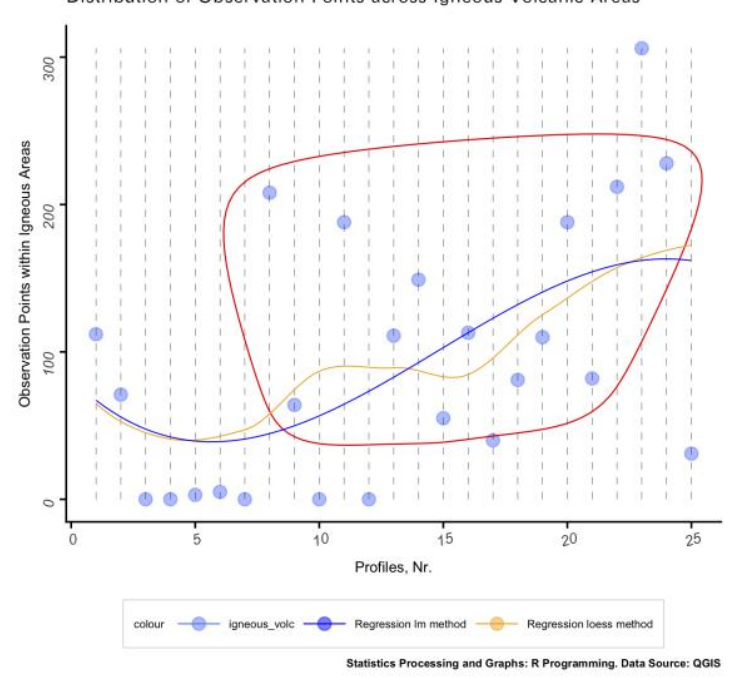

2 马里亚纳海沟。剖面1-25。Mariana Trench, Profiles Nr.1-25. 统计图表。地貌聚类分析, 圆点图。Ranking Dot Plot.
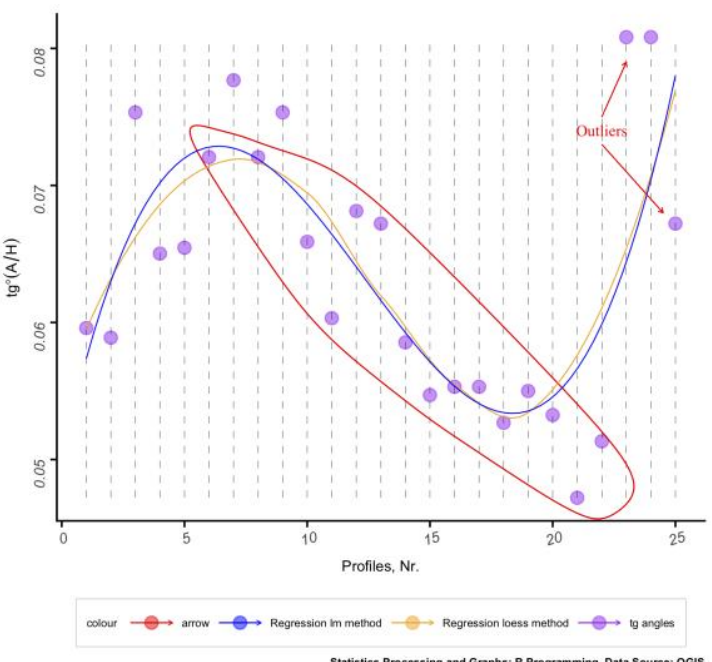

Figure 7. Ranking dot plots by data grouping 
1 Waffle Chart: Mariana Trench

Aspect class of the basement angle

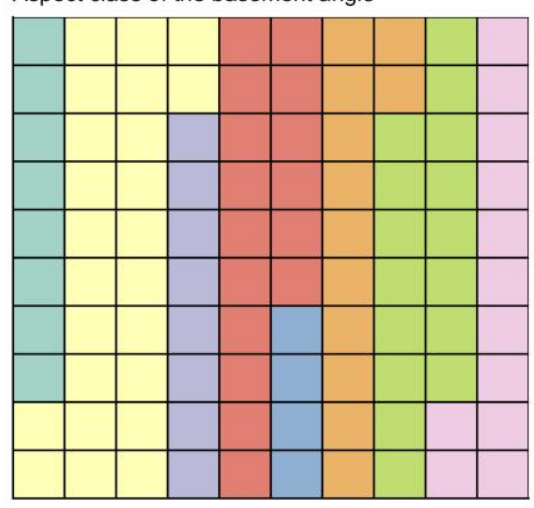

Graphics: R Programming
2 Waffle Chart: Mariana Trench

Steepness angle class of the profiles 1:25

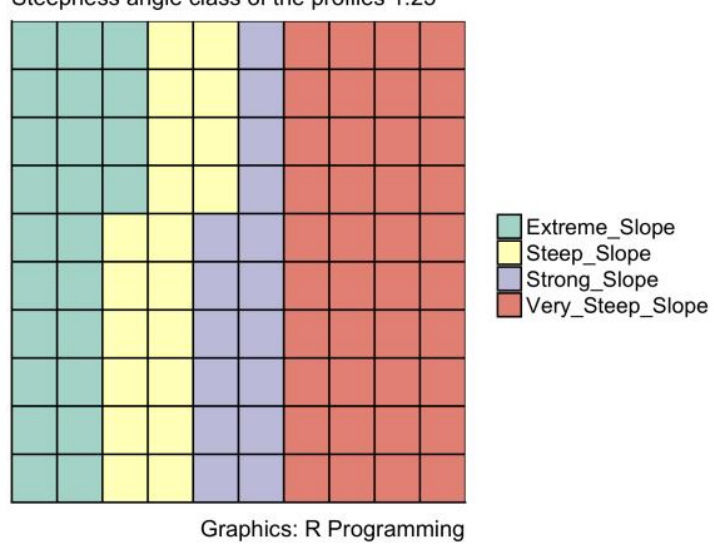

Figure 8. Compositional chart of geometric properties of the Mariana Trench 

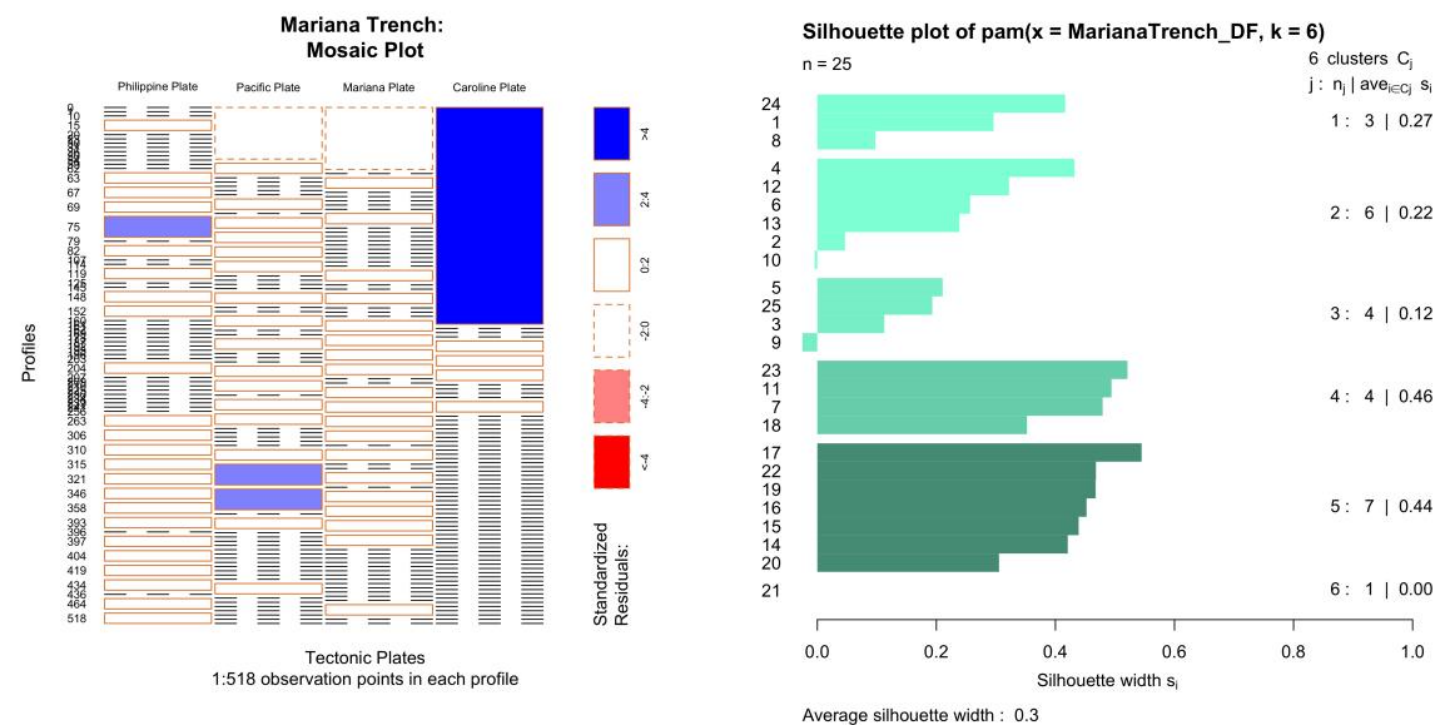

Figure 9. Mosaic plot and silhouette plots for categorical values of the environmental determinants of the Mariana Trench 

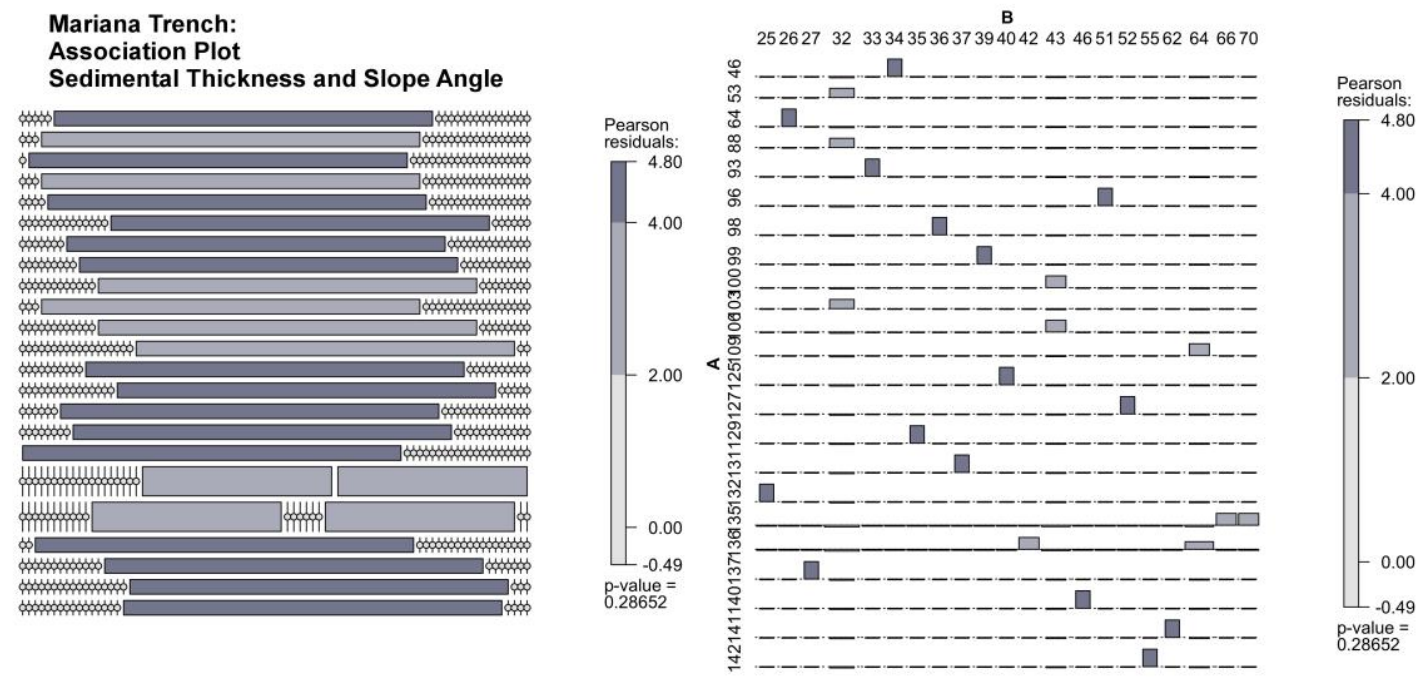

Figure 10. Association plot with statistical Pearson residuals, Mariana Trench 

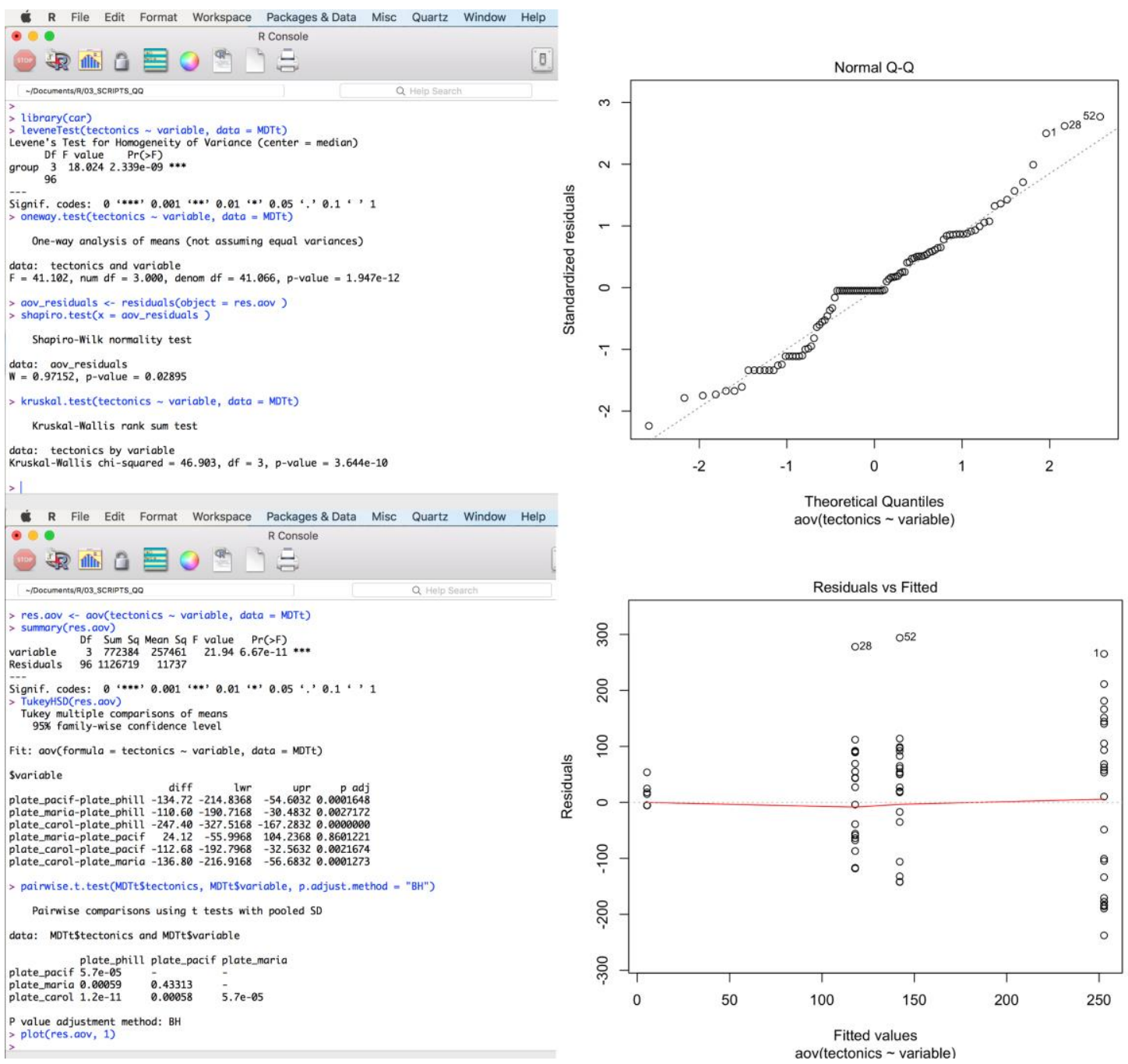

Figure 11. ANOVA hypothesis testing 